\title{
Power Allocation Evaluation for Downlink Non-Orthogonal Multiple Access (NOMA)
}

\author{
Wajd Fahad Alghasmari, Laila Nassef \\ Department of Computer Science \\ Faculty of Computing and Information Technology \\ King Abdulaziz University, Jeddah \\ Saudi Arabia
}

\begin{abstract}
Fifth-generation of wireless cellular systems has the potential to increase capacity, spectral efficiency, and fairness among users. The Non-Orthogonal Multiple Access based wireless networks (NOMA) is the next generation multiplexing technique. NOMA breaks the orthogonality of traditional multiple access to allow multiple users to share the same radio resource simultaneously. The main challenge in designing NOMA is the selection of the resource allocation algorithms since user pairing and power allocation are coupled. This paper compares the performance of three power allocation schemes: fixed power allocation, fractional transmit power allocation and full search power allocation. The algorithms are analyzed in different simulation scenarios using three performance metrics of the spectrum efficiency and energy efficiency and sum rate. Additionally, the impact of user pairing algorithms studied through two user pairing schemes: random user pairing and channel state sorting based user pairing. Results indicate the superiority of NOMA to increase the capacity compared to traditional orthogonal multiple access. On the other hand, full search power allocation is the best performance compared to the other power allocation schemes though it is highly complex compared to fractional transmit power that gives a suboptimal performance.
\end{abstract}

Keywords-Non-Orthogonal Multiple Access; NOMA; Power Allocation; User Pairing; Spectral Efficiency; Sum Rate

\section{INTRODUCTION}

Smartphones nowadays are considered as a core of our lives, due to several services and applications provided, where phone calls are not the only applicable service. Services such as watching TV, playing games, and attending online lectures can be performed easily through our phones. A group of multiple accessing schemes has been introduced over cellular generations to reach this level of service diversity with excellent performance. Multiple accessing schemes that utilized over the past generations have relied on orthogonality that resembles exclusive usage of resources either in time, frequency, or code domain. The first generation has used Frequency Division Multiple Access (FDMA) [1]; the second generation has utilized Time Division Multiple Access (TDMA), and the third generation has used Code Division Multiple Access (CDMA) [2]. The fourth generation Long Term Evolution (LTE), employs Orthogonal Frequency Division Multiple Access (OFDMA) and Single-Carrier (SC)FDMA that have been used as the Orthogonal Multiple Access (OMA) to eliminate mutual interference among users [3].
OMA provides high-performance gain within a reasonable number of users, and it can avoid inter-user interference. Though, OMA suffers from lacking the ability to support an increasing number of users due to the exclusive utilization of the orthogonal resource besides that it cannot provide an excellent experience to all users in the system that causes higher latency and bad cell-edge users' experience [4].

The cellular data traffic is expected to reach a thousandfold for the next decades with respect to the increased number of connected devices in addition to novel technologies integrated, such as the Internet of Things (IoT) [5][6]. Different approaches have been proposed for fifth-generation (5G), one of which is Non-Orthogonal Multiple Access (NOMA), to improve spectral efficiency [7]. In contrast to OMA, NOMA enables concurrent resource sharing among users with negligible interference that results in supporting massive connectivity and boosting spectral efficiency. Accordingly, each user retrieves its signal via complicated Multiuser Detection (MUD) techniques that solve co-channel interference [8]. Moreover, NOMA increases the fairness among users and decrease the latency such that users in cell edge, i.e., low channel quality based on its geographical position can use resources as other users with high channel condition. Unlink OMA, that prefer allocates resources to users with good channel coefficient, which cause delay to users with poor channel condition, which helps in increasing the throughput of cell-edge users [9]. Additionally, NOMA does not have a restriction on the number of users that could be served based on the number of available subchannels [10].

Various non-orthogonal multiple access schemes proposed, such as Multiuser Superposition Transmission (MUST) for LTE [11]. According to [10], NOMA multiplex the users into either power or code domains such as Interleave Division Multiple Access (IDMA), Low Density Spreading (LDS), Sparse Code Multiple Access (SCMA), Pattern Division Multiple Access (PDMA). Power domain NOMA works by superimposing the signals into the same frequency or time domain through Superposition Coding (SC) within distinctive power levels. A superimposed signal that multiplexed over the power domain detected via Successive Interference Cancellation (SIC), which subtracts the interference between signals so that each user can decode its signal at end-users receivers. SIC decodes the coexistence signals iteratively, leading to recovering each user message [12]. The performance of NOMA particularly affected by SIC and the pair of users 
share the same resource [13]. Disparate from OFDMA, channel condition correlated with system performance, where a number of studies stated that a large channel gain difference maximizes the sum rate of the system [14][15].

To support multi-users to share the same time-frequency resources, user pairing and power allocation algorithms are necessary to allocate different power levels. The main concentration of this paper is to compare the performance of three algorithms for resource allocation. The remaining sections of this paper discuss the mathematical model and different user pairing and power allocation algorithms. Section II presents background information of both downlink and uplink NOMA systems, where Section III mathematically presents the computation of the performance metrics of NOMA for the multiple users and multiple subchannels scenario. Section IV presents various user pairing and power allocation schemes. In Section $\mathrm{V}$, the performance scenarios and performance metrics are evaluated to simulate, analyze, and compare performance. Finally, Section VI represents the conclusion and future works.

\section{BACKGROUND}

The principle of NOMA of assigning different power coefficients lies upon both downlink and uplink systems with the difference of where SIC operation is function [16]. SIC process held in the receiver side in downlink NOMA system while uplink NOMA system performs SIC at its transmitter. The design of NOMA is related tightly to the operation of deciding the pair of users to be multiplexed over an individual subchannel and allocating the power levels corresponding to their channel conditions [17]. Paired users in a single subchannel widely suggested to have distinctive channel conditions such that the user with bad channel conditions preferred to pair with the user good channel conditions. Fig. 1 illustrates uplink NOMA system where two users are multiplexed where user1 represents the strong user (i.e., a user with a good channel condition) while user2 represents the weak user (i.e., a user with poor channel condition). The Base Station (BS) in uplink NOMA decodes user1 signal first and subtracts it from the superimposed signal to decode the second user's signal [18]. Therefore, received signal at the base station is represented as:

$y=\sqrt{p_{1}}\left|h_{1}\right| s_{1}+\sqrt{p_{2}}\left|h_{2}\right| s_{2}+v$

such that for user1, $p_{1}$ denotes transmission power, $\left|h_{1}\right|$ represents channel condition between userl and BS, and $s_{1}$ is the transmitted message signal of user1. In contrast, for user2 $\mathrm{p}_{2}$ is the transmitted power and $\left|\mathrm{h}_{2}\right|$ is the channel condition between user2 and BS where $s_{2}$ denote the signal of user2 message. $v$ resemble additive white Gaussian noise (AWGN) in addition to inter-cell interference with spectral density $\mathrm{N}_{0}$ [19].

Downlink NOMA illustrated in Fig. 2, assuming two users and one subchannel and $\left|h_{1}\right|>\left|h_{2}\right|$ which implies UE2 as the weak user and UE1 as the strong user. SIC performed by UE1 that is allocated a low power level to decode UE2 signal and then cancel it to be able to decode its signal at the end [20]. In contrast, UE2 is assigned a high power level and does not have to perform SIC and only decode its signal through treating UE1 signal as interference. Such that assuming $\mathrm{p}_{1}$ and $\mathrm{p}_{2}$ to be the power of the transmitted signal $s_{1}, s_{2}$ for UE1 and UE2, respectively. Moreover, allocated power to both users given as $\mathrm{p}_{1}<\mathrm{p}_{2}$, thus transmitted superimposed signal by $\mathrm{BS}$ is expressed as:

$\mathrm{x}=\sqrt{\mathrm{p}_{1}} \mathrm{~s}_{1}+\sqrt{\mathrm{p}_{2}} \mathrm{~s}_{2}$

where the received signal to the user $\mathrm{i}$ is represented by:

$\mathrm{y}_{\mathrm{i}}=\left|\mathrm{h}_{\mathrm{i}}\right| \mathrm{x}+\mathrm{v}_{\mathrm{i}}$.

Based on Shannon's capacity formula, UE1 and UE2 data rates is represented as [19]:

$\mathrm{R}_{1}=\mathrm{B} \log _{2}\left(1+\frac{\mathrm{p}_{1}\left|\mathrm{~h}_{1}\right|}{\mathrm{N}_{0}}\right)$

$\mathrm{R}_{2}=\mathrm{B} \log _{2}\left(1+\frac{\mathrm{p}_{2}\left|\mathrm{~h}_{2}\right|}{\mathrm{p}_{1}\left|\mathrm{~h}_{2}\right|+\mathrm{N}_{0}}\right)$

Therefore, the capacity of downlink NOMA system with two users is given by the summation of users' data rates in the system as follows:

$\mathrm{R}_{\mathrm{NOMA}}=\mathrm{R}_{1}+\mathrm{R}_{2}$

On the other hand, OFDMA uses the orthogonal multiplexing strategy. In a two users OFDMA system, the bandwidth divided in half where users employ a half by its own, thus the achieved data rates of users given as:

$\mathrm{R}_{1 \mathrm{OMA}}=\frac{\mathrm{B}}{2} \log _{2}\left(1+\frac{\mathrm{p}_{1}\left|\mathrm{~h}_{1}\right|}{\mathrm{N}_{0}}\right)$

$\mathrm{R}_{2 \mathrm{OMA}}=\frac{\mathrm{B}}{2} \log _{2}\left(1+\frac{\mathrm{p}_{2}\left|\mathrm{~h}_{2}\right|}{\mathrm{N}_{0}}\right)$

System's capacity of OMA system is calculated by:

$\mathrm{R}_{\text {OMA }}=\mathrm{R}_{1_{\text {OMA }}}+\mathrm{R}_{2 \text { OMA }}$

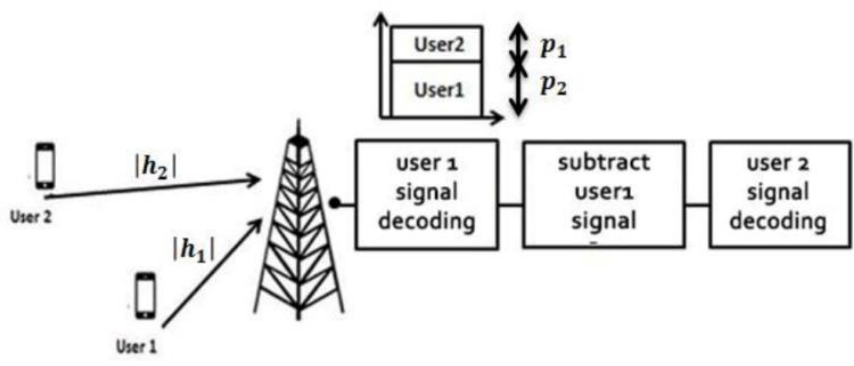

Fig. 1. Uplink Non-Orthogonal Multiple Access.

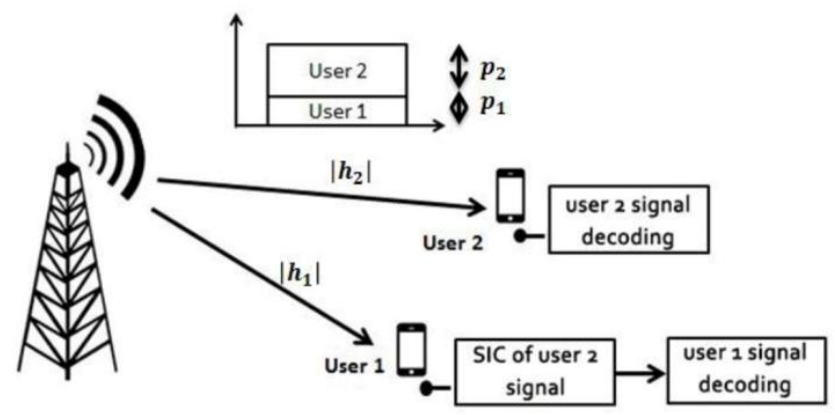

Fig. 2. Downlink Non-Orthogonal Multiple Access. 


\section{DOWnlink Non-OrthogOnAl MultiPle ACCCESS}

A single cell downlink scenario that has a single $\mathrm{BS}$ and $\mathrm{N}$ User Equipment (UEi ) where $i \in N=\{1,2, \ldots, N\}$, and both BS and UE assumed to have one antenna. Overall bandwidth $\mathrm{B}$ is divided into $\mathrm{C}$ subchannels, where the bandwidth of each subchannel is $B_{c}=B / C$. A subset of users $U c=\{U E 1$, $\mathrm{UE} 2, \ldots, \mathrm{UEn}(\mathrm{c})\}$ is assigned to single subchannel $\mathrm{c}$, and the number of multiplexed users over subchannel c limited by 2 users. Therefore, the number of users in the system is $\mathrm{N}=2 \mathrm{C}$. Moreover, the system transmission power is $p_{\text {total }}$ where $p_{i(c)}$ denotes the power level allocated for UEi(c) in subchannel c.

\section{A. Downlink NOMA}

The BS transmits messages to different users over the same subchannel. By that, the message signal $s_{i}$ of UEi superimposed with other users signals multiplexed over the same subchannel, where $E\left[\left|s_{i}\right|^{2}\right]=1$. The superimposed signal transmitted by BS to users at subchannel $\mathrm{c}$ is expressed as:

$\mathrm{x}_{\mathrm{c}}=\sum_{\mathrm{i}=1}^{\mathrm{n}(\mathrm{C})} \sqrt{\mathrm{p}_{\mathrm{i}(\mathrm{c})}} \mathrm{s}_{\mathrm{i}}$

The total transmission power of BS per subchannel is considered to be identical in all subchannels. Hence, the total power is given as $p_{\text {total }}=\sum_{s=1}^{C} P_{s}$ where $P_{s}$ represents the summation of user's power levels in subchannel $c$ which is given by:

$\mathrm{P}_{\mathrm{s}}=\sum_{\mathrm{i}=1}^{\mathrm{n}(\mathrm{c})} \mathrm{p}_{\mathrm{i}(\mathrm{c})}$

On the other side, received signal at UEi(c) over $\left|h_{i(c)}\right|$ that denote the channel gain between the BS and UEi is represented as:

$y_{i(c)}=\left|h_{i(c)}\right| x_{c}+v_{i}$

where the channels gain of all users in each subchannel is sorted as $\left|h_{1}\right|>\left|h_{2}\right|>\cdots>\left|h_{n}\right|[21]$.

In NOMA, SIC decodes the signals iteratively where strong users demultiplex other signals to retrieve its signal in the end where users suffer from bad channel quality decode their signal directly treating other users signals as interference. SIC influenced by power allocation performed by the BS. Therefore, assigned power for users allocated to subchannel c given as $\left|\mathrm{p}_{1(\mathrm{c})}\right|<\left|\mathrm{p}_{2(\mathrm{c})}\right|<\cdots<\left|\mathrm{p}_{\mathrm{i}(\mathrm{c})}\right|$ that means users with poor channel gain given higher power level than users with good channel gain that enhance the fairness in the system. The signal to interference plus noise ratio SINR for UEn(c) in any subchannel $\mathrm{n}$ is given by:

$\operatorname{SINR}_{\mathrm{n}(\mathrm{c})}=\frac{\left|\mathrm{h}_{\mathrm{n}(\mathrm{c})}\right| \mathrm{p}_{\mathrm{n}(\mathrm{c})}}{\left|\mathrm{h}_{\mathrm{n}(\mathrm{c})}\right| \sum_{\mathrm{i}=1}^{\mathrm{n}-1} \mathrm{p}_{\mathrm{i}}(\mathrm{c})+\mathrm{N}_{0}}$

That implies for subchannel c, UEn(c) decode the signals of n-1 users sharing the same subchannel as the weakest user allocated in this subchannel. In contrary the case with UEi(c) that demultiplex and subtract $(\mathrm{UEi}+1(\mathrm{c}), \mathrm{UEi}+2(\mathrm{c}), \ldots$, UEn(c)) message signals while treating stronger user's message signals (UE1(c),..., UEi-2(c), UEi-1(c)) and environmental noise as equivalent noise. Therefore, signal to interference plus noise ratio (SINR) of UEi(c) is expressed as:
$\operatorname{SINR}_{\mathrm{i}(\mathrm{c})}=\frac{\left|\mathrm{h}_{\mathrm{i}(\mathrm{c})}\right| \mathrm{p}_{\mathrm{i}(\mathrm{c})}}{\left|\mathrm{h}_{\mathrm{i}(\mathrm{c})}\right| \sum_{\mathrm{j}=1}^{\mathrm{i}-1} \mathrm{p}_{\mathrm{j}}(\mathrm{c})+\mathrm{N}_{0}}$

For NOMA with multiple users and multiple subchannels, the throughput of UEi(c) is expressed as:

$\mathrm{R}_{\mathrm{i}(\mathrm{c})}=\mathrm{B}_{\mathrm{c}} \log _{2}\left(1+\frac{\left|\mathrm{h}_{\mathrm{i}(\mathrm{c})}\right| \mathrm{p}_{\mathrm{i}(\mathrm{c})}}{\left|\mathrm{h}_{\mathrm{i}(\mathrm{c})}\right| \sum_{\mathrm{j}=1}^{\mathrm{i}-1} \mathrm{p}_{\mathrm{j}}(\mathrm{c})+\mathrm{N}_{0}}\right)$

The sum rate is equivalent to the summation of users rates in subchannel $\mathrm{c}$ that is expressed as:

$\mathrm{R}_{\mathrm{c}}=\sum_{\mathrm{j}=1}^{\mathrm{n}(\mathrm{c})} \mathrm{R}_{\mathrm{j}}$

where the system's sum rate equals the summation of each subchannel and is calculated as:

$\mathrm{R}=\sum_{\mathrm{c}=1}^{\mathrm{C}} \mathrm{R}_{\mathrm{c}}$

Then the spectral efficiency of subchannel $\mathrm{c}$ is defined as the ratio between the sum rate of that subchannel $R_{c}$ and subchannel $c$ bandwidth $B_{c}$ that is represented by [22]:

$\mathrm{SE}_{\mathrm{c}}=\frac{\mathrm{R}_{\mathrm{c}}}{\mathrm{B}_{\mathrm{c}}}$

and the system's spectral efficiency is expressed as:

$\mathrm{SE}=\sum_{\mathrm{c}=1}^{\mathrm{C}} \mathrm{SE}_{\mathrm{c}}$

\section{USER PAIRING AND POWER ALLOCATION}

Users are multiplexed at a single subchannel with different power coefficients. Pairing users is maintained at the transmitter side, such that this pair is assigned to a specific subchannel c. The power allocation determines the power levels for the paired users. The processes of user pairing, as well as the way power allocated among users strictly affect the total sum rate, cell-edge user sum rate, and fairness [23]. Motivated by this fact, this section presents different approaches for power allocation and user pairing.

\section{A. User Pairing}

Generally, two users are multiplexed over the same subchannel in NOMA systems. Though, the number of users able to share an individual subchannel is not limited to two users. That implies the importance of pairing two or more users carefully among a list of available users in the system. To increase the performance of NOMA in terms of the sum rate and decreasing the interference, users with distinctive channel gains are preferred to be grouped over pairing users with similar or adjacent channel gains [24]. Moreover, a user with poor channel conditions is preferred to be paired with a user with high channel conditions. User pairing studied widely were different approaches proposed throughout a verity of researches. In this paper, three users pairing schemes are investigated to attain a clear understanding of the effect of user pairing in the performance.

Generally, two users are multiplexed over the same subchannel in NOMA systems. Though, the number of users able to share an individual subchannel is not limited to two users. That implies the importance of pairing two or more users carefully among a list of available users in the system. To increase the performance of NOMA in terms of the sum rate and decreasing the interference, users with distinctive channel 
gains are preferred to be grouped over pairing users with similar or adjacent channel gains [24]. Moreover, a user with poor channel conditions is preferred to be paired with a user with high channel conditions. User pairing studied widely were different approaches proposed throughout a verity of researches. In this paper, three users pairing schemes are investigated to attain a clear understanding of the effect of user pairing in the performance.

The first is the most straightforward approach that pairs users randomly [25]; it is simple yet inefficient due to the ignorance of the channel states of users. Users having adjacent channel conditions might be paired, which creates significant interference that is caused by assigning similar power levels. The second user pairing is reached through an exhaustive search for all possible pairs of users. This approach has a high computational complexity that grew with the complexity of SIC and signaling overhead [26]. The third scheme is the channel state sorting based user pairing; it is based on ordering users according to their channel conditions. It has low complexity and it can reach an excellent performance [27]. User with the best channel condition is paired with the user with the worst channel condition. Then the following strongest user is grouped with the following weakest user. Therefore, the last pair of users suffer from high interference due to the low channel conditions difference between them. The mechanism of the channel state based sorting algorithm is shown in Fig. 3.

\section{B. Power Allocation}

Power allocation is responsible for assigning different power levels to users sharing the same subchannel. Therefore, the amount of power attributed to a specific subchannel distributed among the users multiplexed over that subchannel. Table I. Provides the comparison between the three power allocation schemes studied in this paper, under the assumption of equivalent subchannel power [19] [28].

- Fixed Power Allocation (FPA): Power is divided between paired users on subchannel $\mathrm{c}$ based on a fixed ratio. Wherever a fraction of subchannel power $\mathrm{P}_{c}$ is allocated to a single user and the remaining power is allocated to the other user, that can be seen as $\left(\alpha \mathrm{P}_{c},\left(1-\alpha \mathrm{P}_{c}\right)\right)$. Despite $\mathrm{P}_{\mathrm{c}}$, the fixed ratio remains uniform for all subbands. Though FPA is low complex, it is considered ineffective due to the inconsideration of users channel conditions in determining power levels.

- Fractional Transmit Power Allocation (FTPA): Similar to uplink LTE power control [29], FTPA used for the multiplexed pair of users, which provides a suboptimal solution. Contradictory to FPA, channel conditions utilized in power allocation such that:

$P_{c, i}=\frac{\left|h_{i}\right|^{-\beta}}{\sum_{j=1}^{n(c)}\left|h_{j}\right|^{-\beta}} P_{c}$

where $\beta$ is fractional quantity of power ranging from 0 to 1 such that if $\beta=0$ an equal power scheme is considered for users pair. Growing in the fractional quantity of power linked to the amount of power allocated to the user with lower channel conditions. The fractional quantity of power $\beta$ fixed in the subchannels. Compared to FPA, FTPA produces higher complexity regarding the increased amount of downlink signaling.

- Full Search Power Allocation (FSPA): In FSPA, power levels of users pair sharing a specific subchannel is given throughout an exhaustive search. This algorithm works by generating all possible set of power levels that reach an optimal solution yet computationally complex. Taking into account a multiplexed pair in subchannel c, all possible set of power levels regarding the channel conditions of each pair is produced leading to choose the best set of power levels based on the performance gain of the system.

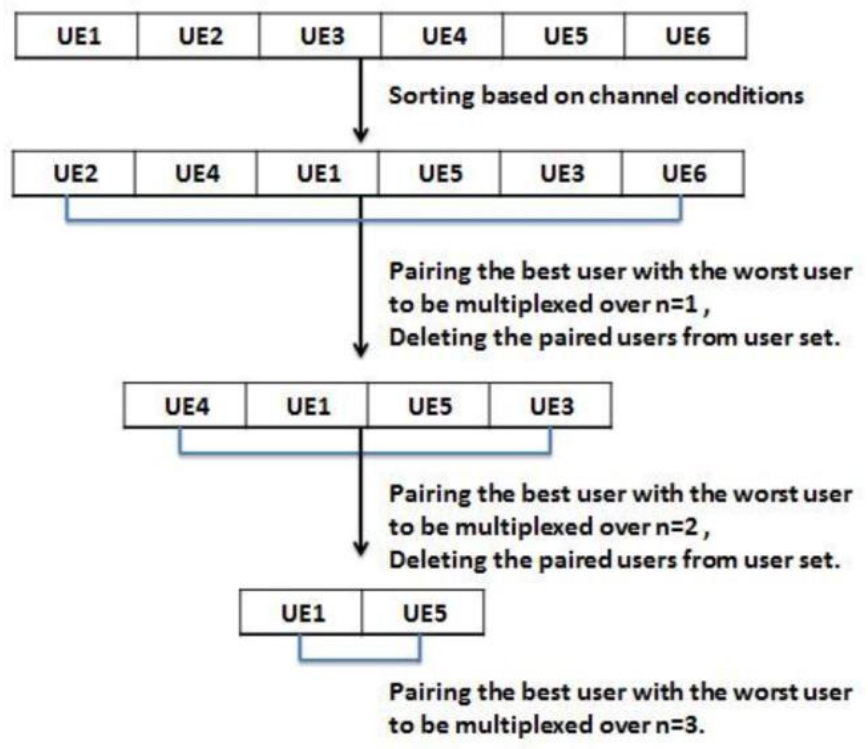

Fig. 3. Channel State based Sorting user Pairing.

TABLE I. COMPARISON OF POWER ALLOCATION SCHEMES

\begin{tabular}{|l|l|l|l|}
\hline & FPA & FTPA & FSPA \\
\hline Scheme type & Static & Dynamic & Dynamic \\
\hline Solution & Suboptimal & Suboptimal & Optimal \\
\hline Advantages & $\begin{array}{l}\text { Decreased } \\
\text { amount of } \\
\text { downlink } \\
\text { signaling }\end{array}$ & $\begin{array}{l}\text { Achieve good } \\
\text { performance with } \\
\text { complexity less } \\
\text { than FSPA }\end{array}$ & $\begin{array}{l}\text { Achieve the best } \\
\text { performance }\end{array}$ \\
\hline Drawbacks & $\begin{array}{l}\text { Cannot satisfy } \\
\text { user's various } \\
\text { QoS requirements }\end{array}$ & $\begin{array}{l}\text { Complexity of } \\
\text { power signaling } \\
\text { compared to FPA }\end{array}$ & $\begin{array}{l}\text { Computationally } \\
\text { complex }\end{array}$ \\
\hline
\end{tabular}

\section{PERformance AnAlysis}

The capacity gain of both NOMA and OFDMA systems versus transmitted power simulated in MATLAB with two users in the system, the channel gains given as $40 \mathrm{~dB}$ and 10 $\mathrm{dB}$ for $\mathrm{UE} 1$ and UE2, respectively. In addition, the power allocation factor for the user with poor channel is 0.75 of the total transmission power. Fig. 4 shows the higher system capacity achieved with NOMA over OFDMA due to the availability of sharing a single subchannel, OMA on the other hand limits the usage of the available bandwidth to a single user which reduce the ability to maximize the usage of available bandwidth that's due to the exclusive usage of the 
available bandwidth to one user. Another consideration from the simulation is that with the increase in the power of transmission, system capacity of both multiple access schemes increased. Allocation of power in NOMA strictly related to SIC process such that a higher power assigned to weak users, which enhances the rates of users under a wide range of channel conditions.

In further simulations, a single cell downlink NOMA assumed with a single BS and multiple users located randomly in the cell, such that the radius of the cell assumed to be $500 \mathrm{~m}$. The BS occupied with one transmitting antenna and users characterized by a single receiver antenna. Transmit power in BS ranged from $10 \mathrm{dBm}$ to $40 \mathrm{dBm}$ where the total bandwidth $\mathrm{B}=5 \mathrm{MHz}$ divided equally over 12 subchannels. The Noise spectral density assumed to be a constant value for all subbands $-150 \mathrm{dBW} / \mathrm{Hz}$. Table II summarize simulation parameters.

\section{A. Numerical Results}

First, we study the effect of the process of assigning different power levels on the performance of downlink NOMA system with four users $(\mathrm{N}=3)$. FPA, FTPA, and FSPA simulated with random user pairing and their performance compared based on system sum rate and spectral efficiency. Power fractional coefficients of FPA and FTPA assumed as $\alpha=0.6$ and $\beta=0.2$. Fig. 5 shows that the sum rate of the system increase with the increase in transmit power for all power allocation schemes. On the other hand, FSPA achieves a higher overall sum rate than both FPA and FTPA, while FTPA performs better than FPA due to the dependability of channel conditions in assigning the power levels, which is not considered in FPA. Though FSPA achieves the best performance, it has higher complexity especially with the increased number of users sharing the same subchannel.

Fig. 6 shows the performance of the system as a function of spectral efficiency versus the transmitted power. The spectral efficiency increases as the transmitted power grow. From the figure, the best performance of the three power allocation schemes reached with the transmitted power is $40 \mathrm{dBm}$. FSPA outperforms the other power allocation schemes due to choosing the best pair of power levels that provide the best performance among the other solutions. Moreover, FTPA performs better than the FPA which can be related to the dynamic nature of power allocation in FTPA.

Furthermore, the relationship between the number of users and the system's sum rate studied for two user pairing schemes. Random user pairing and channel state sorting based user pairing simulated with FPA, the system assumed to serves numbers of users up to 24 users per cell where the transmission power assumed to be equal $40 \mathrm{dBm}$. Fig. 7 represents the behavior of the overall sum rate concerning the variation of the number of users served. The result shows that with the increase in the number of users served, the sum rate increases as a response. Additionally, channel state sorting based user pairing maintain slightly higher performance than random user pairing due to the utilization of channel conditions where the users paired with a higher difference in their channel conditions though the effect of pairing users with highly distinctive channel conditions is minimal. On the other hand, the gain difference between these two algorithms appeared with a larger number of users served in the cell, such that with less than 8 users served the performance of both schemes is equivalent.

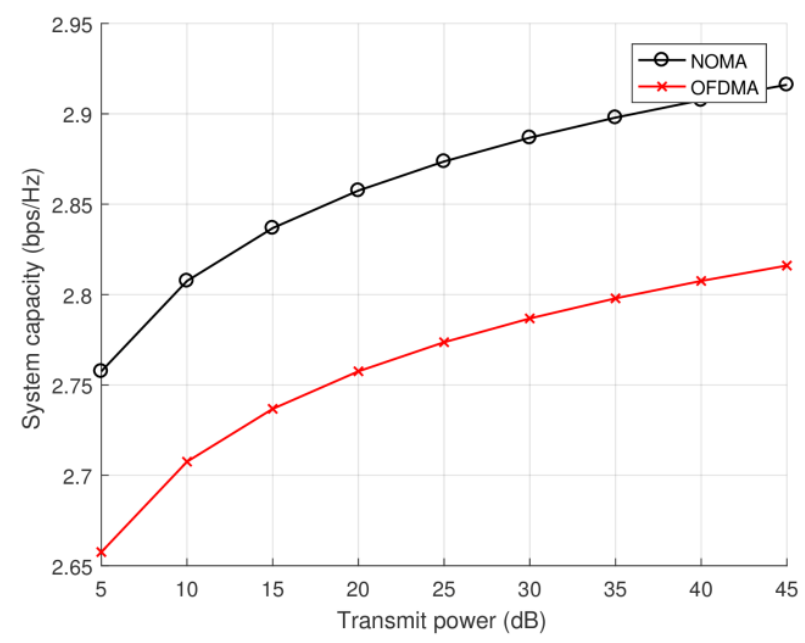

Fig. 4. Capacity Gain of NOMA and OFDMA.

TABLE II. SimUlation PARAMETERS

\begin{tabular}{|l|l|}
\hline Parameters & Values \\
\hline Cell radius & $500 \mathrm{~m}$ \\
\hline Transmitted power & $10 \mathrm{~W}(40 \mathrm{dBm})$ \\
\hline Total Bandwidth & $5 \mathrm{MHz}$ \\
\hline Number of resource blocks $(\mathrm{RP})$ & 24 \\
\hline Number of subchannels & 12 \\
\hline Noise Spectral Density & $-150 \mathrm{dBw} / \mathrm{Hz}$ \\
\hline Channel estimation & Ideal \\
\hline Channel & AWGN \\
\hline Traffic Model & Full Buffer \\
\hline Number of multiplexed users & 2 \\
\hline Maximum Number of users & 24 \\
\hline Number of transmit antenna at BS & 1 \\
\hline Number of receiver antenna at UE & 1 \\
\hline
\end{tabular}

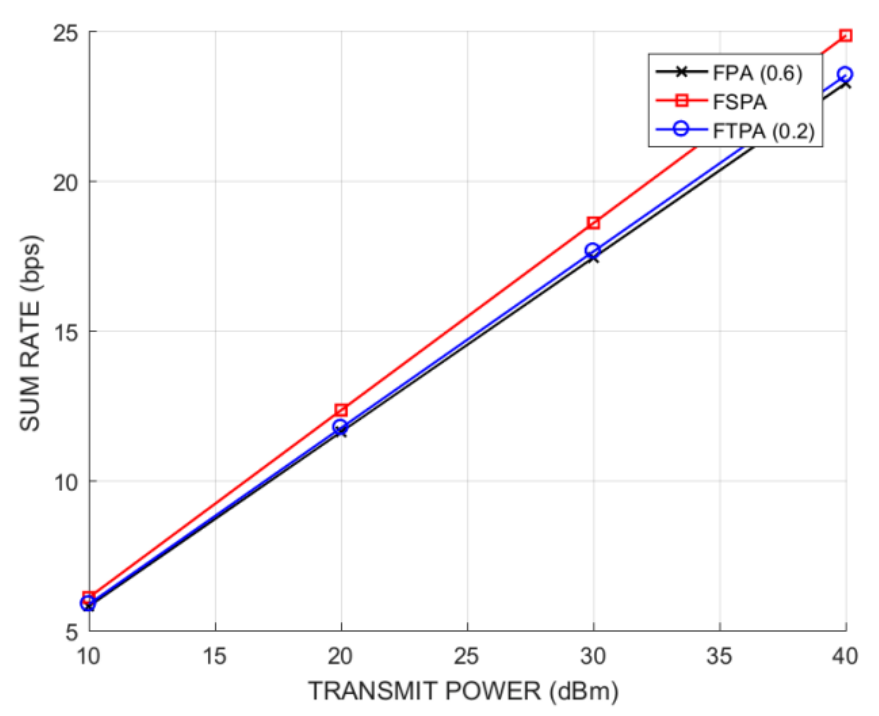

Fig. 5. Sum Rate of different Power Allocation Algorithms. 


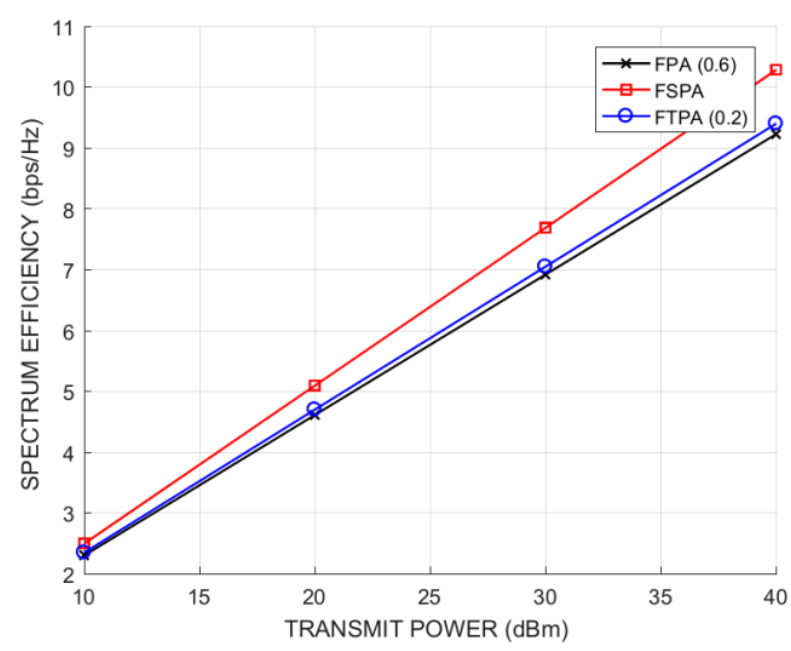

Fig. 6. Spectral Efficiency of different Power Allocation Algorithms.

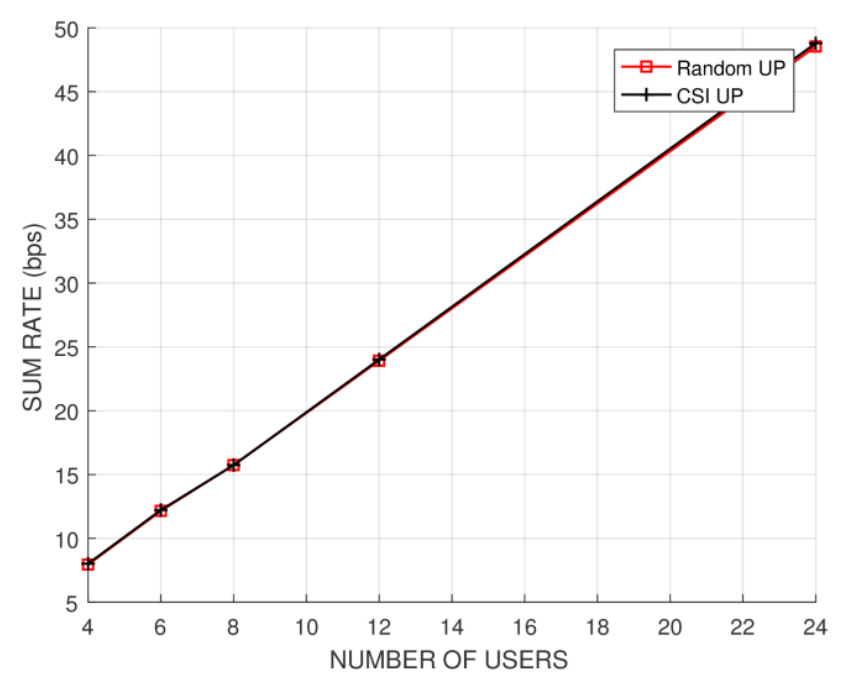

Fig. 7. Sum Rate of different user Pairing Algorithms.

\section{CONCLUSION AND FutURE WORKS}

The next-generation cellular system demands highly effective technologies to be adopted to understand the new services; one of the proposed technologies is NOMA. The fundamental working principle of NOMA is the new power domain, along with the frequency and time domains. Resource allocation is investigated in downlink NOMA with multiple numbers of users such as FSPA, FTPA, and FPA. On the other hand, random user pairing and channel state sorting based user pairing are studied. The simulation indicated a higher capacity gain of NOMA over traditional OMA. In addition, FSPA has obtained superior performance than FPA and FTPA. On the other hand, the results have revealed a little effect on the system through pairing users with significant differences in channel conditions. Future research should be devoted to the development of a low complex power allocation algorithm using the concept of heuristic search.

\section{REFERENCES}

[1] Faruque, S. (2019). Frequency Division Multiple Access (FDMA). In Radio Frequency Multiple Access Techniques Made Easy. Springer, Cham. https://doi.org/https://doi.org/10.1007/978-3-319-91651-4_3
[2] Bawazir, S. S., Sofotasios, P. C., Muhaidat, S., Al-Hammadi, Y., \& Karagiannidis, G. K. (2018). Multiple Access for Visible Light Communications: Research Challenges and Future Trends. IEEE Access, 6, 26167-26174. https://doi.org/doi: 10.1109/ACCESS.2018. 2832088

[3] Kalhoro, S., Umrani, F. A., Khanzada, M. A., \& Rahoo, L. A. (2019). Matched Filter Based Spectrum Sensing Technique for 4G Cellular Network. Mehran University Research Journal of Engineering and Technology, 38, 973-978. https://doi.org/https://doi.org/10.22581 /muet1982.1904.10

[4] Wei, Z., Yuan, J., Ng, D. W. K., Elkashlan, M., \& Ding, Z. (2016). A Survey of Downlink Non-orthogonal Multiple Access for 5G Wireless Communication Networks. ZTE Communications, 14(4). https://doi.org/10.3969/j. issn. 1673-5188

[5] Andrews, J. G., Buzzi, S., Choi, W., Hanly, S. V., Lozano, A., Soong, A. C. K., \& Zhang, J. C. (2014). What will 5G be? EEE Journal on Selected Areas in Communications, 32(6), 1065-1082. https://doi.org/ 10.1109/JSAC.2014.2328098

[6] Li, Q. C., Niu, H., Papathanassiou, A. T., \& Wu, G. (2014). 5G network capacity: Key elements and technologies. IEEE Vehicular Technology Magazine, 9(1), 71-78. https://doi.org/10.1109/MVT.2013.2295070

[7] Agyapong, P. K., Iwamura, M., Staehle, D., Kiess, W., \& Benjebbour, A. (2014). Design considerations for a $5 \mathrm{G}$ network architecture. IEEE Communications Magazine, 52(11), 65-75. https://doi.org/10.1109/ MCOM.2014.6957145

[8] Wu, Z., Lu, K., Jiang, C., \& Shao, X. (2018). Comprehensive Study and Comparison on 5G NOMA Schemes. IEEE Access, 6, 18511-18519. https://doi.org/10.1109/ACCESS.2018.2817221

[9] Saito, Y., Kishiyama, Y., Benjebbour, A., Nakamura, T., Li, A., \& Higuchi, K. (2013). Non-orthogonal multiple access (NOMA) for cellular future radio access. In 2013 IEEE 77th Vehicular Technology Conference (VTC Spring) (pp. 1-5). Dresden. https://doi.org/10.1109/VTCSpring.2013.6692652

[10] Dai, L., Wang, B., Yuan, Y., Han, S., I, C., \& Wang, Z. (2015). Nonorthogonal multiple access for 5G: solutions, challenges, opportunities, and future research trends. IEEE Communications Magazine, 53(9), 7481. https://doi.org/10.1109/MCOM.2015.7263349

[11] Lee, H., Kim, S., \& Lim, J.-H. (2016). Multiuser Superposition Transmission (MUST) for LTE-A systems. In 2016 IEEE International Conference on Communications (ICC) (pp. 1-6). Kuala Lumpur. https://doi.org/10.1109/ICC.2016.7510909

[12] Li, G. Y., Niu, J., Lee, D., Fan, J., \& Fu, Y. (2014). Multi-cell coordinated scheduling and MIMO in LTE. IEEE Communications Surveys \& Tutorials, 16(2), 761-775. https://doi.org/10.1109/ SURV.2014.022614.00186

[13] Choi, J. (2018). Throughput analysis for multiuser diversity of two users with SIC in NOMA systems. In 2018 International Conference on Signals and Systems (ICSigSys) (pp. 120-124). Bali. https://doi.org/10.1109/ICSIGSYS.2018.8372649

[14] Murti, F. W., Siregar, R. F., \& Shin, S. Y. (2018). Exploiting nonorthogonal multiple access in downlink coordinated multipoint transmission with the presence of imperfect channel state information. Information Theory.

[15] Yuan, Y., Yuan, Z., Yu, G., Hwang, C., Liao, P., Li, A., \& Takeda, K. (2016). Non-orthogonal transmission technology in LTE evolution. IEEE Communications Magazine, 54(7), 68-74. https://doi.org/10.1109/MCOM.2016.7509381

[16] Benjebbour, A., Saito, Y., Kishiyama, Y., Li, A., Harada, A., \& Nakamura, T. (2013). Concept and practical considerations of nonorthogonal multiple access (NOMA) for future radio access. In 2013 International Symposium on Intelligent Signal Processing and Communication Systems (pp. 770-774). Naha. https://doi.org/10.1109/ ISPACS.2013.6704653

[17] Eddo, Z., Hojeij, M.-R., Nour, C. A., Farah, J., \& Douillard, C. (2016). Evaluation of Intra-Subband Power Allocation for a Downlink NonOrthogonal Multiple Access (NOMA) System. In 2016 IEEE Globecom Workshops (GC Wkshps) (pp. 1-7). Washington, DC. https://doi.org/10.1109/GLOCOMW.2016.7848899 
[18] Al-Imari, M., Xiao, P., Imran, M. A., \& Tafazolli, R. (2014). Uplink non-orthogonal multiple access for 5G wireless networks. In 2014 11th International Symposium on Wireless Communications Systems (ISWCS) (pp. 781-785). Barcelona. https://doi.org/10.1109/ISWCS. 2014.6933459

[19] BENJEBBOUR, A., \& HIGUCHI, K. (2015). Non-orthogonal Multiple Access (NOMA) with Successive Interference Cancellation for Future Radio Access. IEICE TRANSACTIONS on Communication, E98-B(3), 403-414. https://doi.org/10.1587/transcom.E98.B.403

[20] Yan, C., Harada, A., Benjebbour, A., Lan, Y., Li, A., \& Jiang, H. (2015). Receiver Design for Downlink Non-Orthogonal Multiple Access (NOMA). In 2015 IEEE 81st Vehicular Technology Conference (VTC Spring) (pp. 1-6). Glasgow. https://doi.org/10.1109/VTCSpring.2015. 7146043

[21] Kassir, A., Dziyauddin, R. A., Kaidi, H. M., \& Izhar, M. A. M. (2018). A Review of Power Domain Non-Orthogonal Multiple Access in 5G Networks. International Journal of Integrated Engineering, 10(7), 253262. https://doi.org/https://doi.org/10.30880/ijie.2018.10.07.023

[22] Bizaki, H. K. (2016). Towards 5G Wireless Networks: A Physical Layer Perspective. https://doi.org/10.5772/63098

[23] Li, A., Lan, Y., Chen, X., \& Jiang, H. (2015). Non-orthogonal multiple access (NOMA) for future downlink radio access of 5G. China Communications, 12(Supplement), 28-37. https://doi.org/10.1109/ CC. 2015.7386168
[24] Ding, Z., Yang, Z., Fan, P., \& Poor, H. V. (2014). On the performance of non-orthogonal multiple access in 5G systems with randomly deployed users. IEEE Signal Processing Letters, 21(12), 1501-1505. https://doi.org/10.1109/LSP.2014.2343971

[25] Ding, Z., Fan, P., \& Poor, H. V. (2015). User pairing in non-orthogonal multiple access downlink transmissions. In 2015 IEEE Global Communications Conference (GLOBECOM). San Diego. https://doi.org/10.1109/GLOCOM.2015.7417061

[26] Islam, S. M. R., Zeng, M., Dobre, O. A., \& Kwak, K.-S. (2018). Resource allocation for downlink NOMA systems: Key techniques and open issues. IEEE Wireless Communications, 25(2), 40-47. https://doi.org/10.1109/MWC.2018.1700099

[27] Ding, Z., Fan, P., \& Poor, H. V. (2015). Impact of User Pairing on 5G Nonorthogonal Multiple-Access Downlink Transmissions. IEEE Transactions on Vehicular Technology, 65(8), 6010-6023. https://doi.org/10.1109/TVT.2015.2480766

[28] Benjebbovu, A., Li, A., Saito, Y., Kishiyama, Y., Harada, A., \& Nakamura, T. (2013). System-level performance of downlink NOMA for future LTE enhancements. In 2013 IEEE Globecom Workshops (GC Wkshps). Atlanta. https://doi.org/10.1109/GLOCOMW.2013.6824963

[29] Endo, Y., Kishiyama, Y., \& Higuchi, K. (2012). Uplink non-orthogonal access with MMSE-SIC in the presence of inter-cell interference. In 2012 International Symposium on Wireless Communication Systems (ISWCS). Paris: IEEE. https://doi.org/10.1109/ISWCS.2012.6328370. 